\title{
HARMONIC FUNCTIONS AND ENDS OF GRAPHS
}

\author{
by MASSIMO A. PICARDELLO and WOLFGANG WOESS
}

(Received 3rd February 1987)

\section{Introduction}

In this note we discuss extensions of results [5], where transient random walks are considered, whose transition matrix is compatible with a tree-structure of the underlying discrete state space. Notation is generally as in [5], with the exception that instead of a tree $T$ we consider an arbitrary graph $\Gamma$, which is locally finite, connected, and whose vertex set $S$ is infinite. The edge set $E$ is unoriented, there are no multiple edges. We consider a transition operator $P$, given by a stochastic matrix $(p(u, v))_{u, v \in S}$, which gives rise to a transient Markov chain ("random walk"), related to the graph structure by:

(i) if $p(u, v)>0$ then $[u, v] \in E$,

(ii) there is an $M>0$ such that $p^{(k)}(u, v)>0$ for some $k=k(u, v) \leqq M$ whenever $[u, v] \in E$ ("uniform irreducibility").

Note that (i) yields no restriction: we can always redefine the edges so that this condition holds.

We are interested in the cone $\mathrm{H}^{+}$of positive harmonic functions, i.e. the functions $h$ on $S$ satisfying

$$
\sum_{v} p(u, v) h(v)=h(u) \quad \text { for every } u \text { in } S .
$$

Recall that the Martin kernel is defined by

$$
K(u, v)=F(u, v) / F(e, v), \quad u, v \in S,
$$

where $e$ is a fixed reference vertex and $F(u, v)$ is the probability that the random walk, starting at $u$, reaches $v$ at some instant $n \geqq 0$. The Martin compactification $\hat{S}$ is the smallest compactification of $S$ to which $K$ extends continuously in the second variable, and $S^{*}=\widehat{S} \backslash S$ is the Martin boundary. By (i), each row of the transition matrix has only finitely many nonzero entries. Hence $K(\cdot, x)$ is in $H^{+}$for every $x \in S^{*}$, and if $h \in H^{+}$, then the Poisson-Martin representation theorem says that

$$
h=\int_{S^{*}} K(\cdot, x) v_{h}(d x)
$$


for some positive Borel measure $v_{h}$ on $S^{*}$. The reader is referred to [5] for a more detailed exposition of the preliminaries and for references in addition to those given below.

We intend to provide conditions under which the Martin boundary coincides (by homeomorphism) with the space of ends of $\Gamma$. Proofs will be only outlined, as they are direct extensions of those in [5].

\section{The space of ends}

Following [3] and [4], we give a "combinatorial" description of the space of ends of $\Gamma$, originally introduced in [2].

A (simple) path in $\Gamma$ is a finite or one-sided infinite sequence of successively contiguous vertices (without repetitions). The distance $d(u, v)$ of two vertices is the length (number of edges) of the shortest path between $u$ and $v$. Two infinite simple paths are equivalent if, for any finite $U \subset S$, they can be connected by some finite path lying outside $U$. An end of $\Gamma$ is an equivalence class under this relation. The set of all ends is denoted by $\Omega$. We endow $S \cup \Omega$ with a topology: if we remove a finite $U \subset S$, then $\Gamma$ splits into finitely many connected components. We include into each of these components also all ends which can be represented by some infinite simple path lying entirely within the component. In this sense, if $x \in(S \backslash U) \cup \Omega$, then $C(U, x)$ denotes the component containing $x$. Now a basis of the topology on $S \cup \Omega$ is given by all sets $C(U, x)$ which are obtained in this way from all finite $U \subset S$. This makes $S \cup \Omega$ a totally disconnected, compact Hausdorff space which contains $\Omega$ as a compact subspace and $S$ as a discrete, dense subset.

Definition. We say that a sequence $\left\{U_{n}\right\}$ of finite subsets of $S$ is contracting towards $\omega \in \Omega$, if

(1) $C\left(U_{n-1}, \omega\right) \supset U_{n} \cup C\left(U_{n}, \omega\right)$, and

(2) $\left\{C\left(U_{n}, \omega\right)\right\}$ constitutes a neighbourhood basis at $\omega$.

Such a sequence exists for every $\omega \in \Omega$ by the way the topology is defined.

\section{Results}

Define $F_{W}(u, v)$ to be the probability that the random walk, starting at $u$, reaches $v \in S$ before hitting $W \subset S$. The following result can be obtained as a corollary of [7, Theorem 5 and Corollary 4]. We indicate a "discrete" approach to the proof.

Proposition. The identity on $S$ extends to a continuous surjection $\hat{S} \rightarrow S \cup \Omega$, which maps $S^{*}$ onto $\Omega$.

Proof. By local finiteness, $S$ is discrete in $\hat{S}$. By compactness of $\hat{S}$ and $S \cup \Omega$ it is enough to prove the following: if $\left\{u_{n}\right\},\left\{v_{n}\right\}$ are sequences in $S$, converging to $x \in S^{*}$, and $u_{n} \rightarrow \omega, v_{n} \rightarrow \omega^{\prime}$ for $\omega, \omega^{\prime} \in \Omega$, then $\omega=\omega^{\prime}$.

Assume $\omega \neq \omega^{\prime}$. Then there is a ball $B_{N}=\{w \in S \mid d(e, w) \leqq N\}$ such that 
$d\left(C\left(B_{N}, \omega\right), C\left(B_{N}, \omega^{\prime}\right)\right)>M . \quad$ Set $\quad U=B_{N+1} \cap C\left(B_{N}, \omega\right), \quad V=B_{N+1} \cap C\left(B_{N}, \omega^{\prime}\right) . \quad$ As $d(U, V)>M, F_{V}\left(u, u^{\prime}\right)>0$ for $u, u^{\prime} \in U$ and $F_{v}\left(v, v^{\prime}\right)>0$ for $v, v^{\prime} \in V$ : this follows from assumptions (i) and (ii). If $Q_{v}$ and $Q_{V}$ denote the "first hitting" operators of $U$ and $V$ $[5, \S 3]$, and $f_{n}=K\left(\cdot, u_{n}\right), g_{n}=K\left(\cdot, v_{n}\right) f=\lim f_{n}=\lim g_{n}$, then

$$
f_{n}=Q_{U} f_{n} \text { on } V \text { and } g_{n}=Q_{V} g_{n} \text { on } U \text { for large } n
$$

In the limit, we obtain

$$
f=Q_{U} Q_{V} f \text { on } V \text { and } f=Q_{V} Q_{U} f \text { on } U .
$$

Now [5, Proposition 1] applies and yields a contradiction.

Next, we exhibit a condition, under which the above surjection becomes a homeomorphism. For $U \subset S$, define

$\tilde{U}=\{u \in S \mid d(u, v) \leqq M / 2$ for some vertex $v$ on a shortest path between two points of $U\}$.

Theorem. Suppose that for each $\omega \in \Omega$ there is a sequence $\left\{U_{n}\right\}$ of finite sets contracting towards $\omega$, such that $\tilde{U}_{n} \cap U_{n+1}=\emptyset$ for all $n$, and

$$
\sum_{n} \min \left\{F_{U_{n+1}}(u, v) \mid u, v \in U_{n}\right\}=\infty .
$$

then (a) the Martin boundary $S^{*}$ coincides with the space $\Omega$ of all ends of $\Gamma$, and (b) all ends are extreme points of the boundary.

Proof. To prove (a), in view of the Proposition we must show that for every $u \in S$, $\left\{K\left(u, v_{n}\right)\right\}$ converges, if $\left\{v_{n}\right\}$ converges to some end $\omega$, and that the limit is independent of the particular choice of this sequence. This can be done in the same way as in [5, 84$]$, once an equivalent of $[5$, Theorem 1$]$ has been established in the present setting.

For $n \in \mathbb{N}$, consider the "intermediate" matrix $A_{n}=\left(\alpha_{n}(u, v)\right)_{u \in U_{n}, v \in U_{n+1}}$, where $\alpha_{n}(u, v)=$ $Q_{U_{n+1}} \delta_{v}(u)$, see $[5, \S 3]$. Its zeros are disposed in columns (as $\left.\tilde{U}_{n} \cap U_{n+1}=\emptyset\right)$, and by $[6, \S 3.1]$ and [1], its Birkhoff contraction coefficient is

$$
\tau\left(A_{n}\right)=\frac{1-\sqrt{\Phi\left(A_{n}\right)}}{1+\sqrt{\Phi\left(A_{n}\right)}} \text {, with } \Phi\left(A_{n}\right)=\min \frac{\alpha_{n}(u, v) \alpha_{n}\left(u^{\prime}, v^{\prime}\right)}{\alpha_{n}\left(u^{\prime}, v\right) \alpha_{n}\left(u, v^{\prime}\right)},
$$

where the minimum ranges over all nonnegative entries of $A_{n}$. Now, convergence of $\left\{K\left(u, v_{n}\right)\right\}$ will follow from projective convergence of the matrix products $A_{1} A_{2} \ldots A_{n}$, as $n \rightarrow \infty$. The latter holds if $\tau\left(A_{1} \ldots A_{n}\right) \rightarrow 0$, which in turn is true if $\sum_{n} \sqrt{\Phi\left(A_{n}\right)}=\infty$. (In [6, Theorem 3.2], this is stated for "allowable" square matrices but carries over to the present setting as in [5].) Now it is easy to see that

$$
\alpha_{n}(u, v) / \alpha_{n}\left(u^{\prime}, v\right) \geqq F_{U_{n+1}}\left(u, u^{\prime}\right) \text { for } u, u^{\prime} \in U_{n}, v \in U_{n+1},
$$


so that

$$
\Phi\left(A_{n}\right) \geqq\left(\min \left\{F_{U_{n+1}}\left(u, u^{\prime}\right) \mid u, u^{\prime} \in U_{n}\right\}\right)^{2} .
$$

This proves (a), and (b) is proved exactly as in [5, Proposition 4].

If $f$ is any real function of the finite subsets of $S$, then $\liminf _{U \omega_{\omega} \omega} f(U)$ denotes the infimum among all numbers $\liminf _{n} f\left(U_{n}\right)$, taken over all sequences $\left\{U_{n}\right\}$ of finite sets contracting to $\omega \in \Omega$. In this sense,

$$
\operatorname{diam}(\omega)=\liminf _{U \varsigma \omega} \operatorname{diam}(U) \text { and } \delta(\omega)=\liminf _{U_{\iota} \omega} \delta(\omega),
$$

where $\delta(U)=\min \{p(u, v)>0 \mid u, v \in \tilde{U}\}, \delta(U)=1$ if $\# U=1$.

Corollary. Each of the following conditions implies statements (a) and (b) of the Theorem:

(I) For each $\omega \in \Omega$ there is a sequence $\left\{U_{n}\right\}$ contracting towards $\omega$, such that $\tilde{U}_{n} \cap U_{n+1}=\emptyset$ and

$$
\sum_{n} \delta\left(U_{n}\right)^{M \operatorname{diam}\left(U_{n}\right)}=\infty
$$

(II) For each $\omega \in \Omega, \operatorname{diam}(\omega)<\infty$ and $\delta(\omega)>0$.

As an application of (I), consider the following example: let $S$ be the set of integers, equipped with edges $[n, n+1]$ and $[n,-n], n \in \mathbb{Z}$ (Fig. 1 ), $p(i, j)>0$ iff $[i, j]$ is an edge.

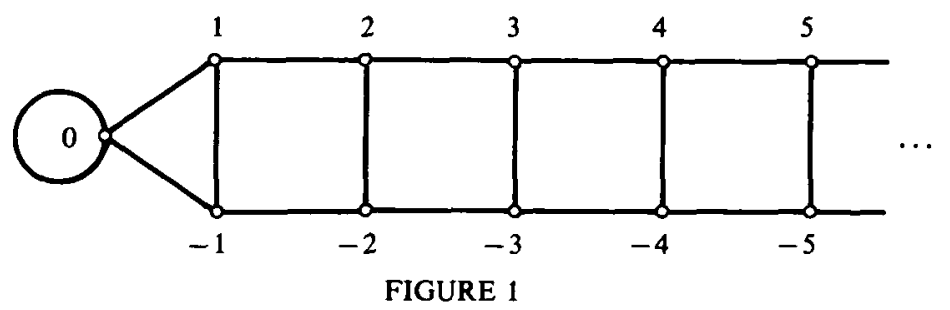

This graph has only the end $\omega=\infty$, and the sets $U_{n}=\{-n, n\}, n \in \mathbb{N}$ contract towards $\infty$. Furthermore $\tilde{U}_{n}=U_{n}, \operatorname{diam}\left(U_{n}\right)=1$, and $\delta\left(U_{n}\right)=\min \{p(n,-n), p(-n, n)\}$. If the random walk is transient and $\sum \delta\left(U_{n}\right)=\infty$, then the Martin boundary consists of the single point $\infty$, that is, all positive harmonic functions are constant. On the other hand, it is easy to construct examples where $\delta\left(U_{n}\right)$ decreases exponentially and there are nonconstant functions in $H^{+}$.

Condition (II) applies, for example, for the (transient) simple random walk on a graph $\Gamma$, whose vertices have bounded degree, and whose ends all have finite diameter. One may ask which finitely generated groups have Cayley graphs with the latter property: they are just the finite extensions of free groups [8]. 


\section{REFERENCES}

1. G. Birkhoff, Extensions of Jentzsch's theorem, Trans. Amer. Math. Soc. 85 (1957), 219-227.

2. H. Freudenthal, Über die Enden diskreter Räume und Gruppen, Comment. Math. Helv. 17 (1944), 1-38.

3. R. Halin, Über unendliche Wege in Graphen, Math. Ann. 157 (1964), 125-137.

4. H. A. Jung, Connectivity in infinite graphs, Studies in Pure Math. (Ed. L. Mirsky, Academic Press, 1971), 137-143.

5. M. A. Picardello and W. Woess, Martin boundaries of random walks: Ends of trees and groups, Trans. Amer. Math. Soc. 302 (1987), 185-205.

6. E. Seneta, Non-Negative Matrices and Markov Chains, 2nd ed. (Springer, 1981).

7. J. C. TAYLor, The Martin boundaries of equivalent sheaves, Ann. Inst. Fourier 20 (1970), 433-456.

8. W. WoEss, Graphs and groups with tree-like properties, (1986), preprint.

Dipartimento di Matematica "Guido Castelnuovo"

UNIVERSITA DI ROMA

I-00100 ROMA

ITALY

Current Address (M. A. Picardello):

Dipartimento di Matematica

UNIVERSITÁ DELL'AQUILA

I-67100 L'AQUILA

ITALY
INSTITUT FUR MATHEMATIK UND Angewandte Geometrie MONTANUNIVERSITÄT A-8700 LEOBEN

Austria 